\title{
Priority of the Specific Epithet anguillimortiferum over the Specific Epithet tarda in the Name of the Organism Presently Known as Edwardsiella tarda
}

\author{
R. SAKAZAKI AND K. TAMURA \\ Department of Bacteriology 1, National Institute of Health, Kamiosaki, Shinagawa-ku, Tokyo, Japan
}

Hoshina, in 1962, described a new species, Paracolobacterum anguillimortiferum. A comparison of the characteristics given in the original description by Hoshina of the new bacterium with those of Edwardsiella tarda Ewing and McWhorter 1965 indicates that these organisms are identical. Although the original strain of $P$. anguillimortiferum was lost, the name was validly published, and therefore the specific epithet "anguillimortiferum" has priority over "tarda." The new combination Edwardsiella anguillimortifera is proposed for this organism. The type strain of Edwardsiella tarda ATCC 15947 is designated as the neotype strain of Edwardsiella anguillimortifera.

The scientific name Edwardsiella tarda Ewing and McWhorter 1965 (1) was given to a group of organisms with several vernacular names, including Asakusa (4) and Bartholomew (3). The name Edwardsiella tarda has now been commonly accepted. Recently, this organism has attracted attention as the causative agent of a disease in eels. Although several other species of gram-negative rods have been reported as the cause of disease in eels, we studied the organism described by Hoshina (2) for reasons of taxonomy. Hoshina placed the organism in the genus Paracolobactrum and proposed for it the name Paracolobactrum anguillimortiferum. He described this organism as follows: "Gram-negative, asporogenous, motile rods with peritrichous flagella; facultatively anaerogenic; acapsulated; gelatin was not liquefied; methyl red positive; Voges-Proskauer negative, nitrates were reduced to nitrites: indole and hydrogen sulfide were produced; acid and gas in glucose; fructose, galactose and maltose; no acid and gas in cellobiose, lactose, raffinose, rhamnose, sorbose, trehalose, xylose, dulcitol, mannitol, sor- bitol, sucrose, glycogen, starch, inulin, salicin and esculun; nicotic acid was required as a growth factor."

The characteristics of Paracolobactrum anguillimortiferum as given by Hoshina are compared in Table 1 with the characteristics of over 800 strains of Edwardsiella tarda, including the type strain, ATCC 15947.

It is clear from the table that the characteristics of $P$. anguillimortiferum are identical with those of Edwardsiella tarda. Unfortunately, the original strain of Paracolobactrum anguillimortiferum on which Hoshina based his description has been lost, but the name was validly published by Hoshina, and the specific epithet "anguillimortiferum" therefore has priority over the specific epithet "tarda" in the name Edwardsiella tarda Ewing and McWhorter. Therefore we propose Edwardsiella anguillimortifera (Hoshina) comb. nov. to replace the illegitimate combination Edwardsiella tarda. The type strain of Edwardsiella tarda ATCC 15947 is designated as the neotype strain of Edwardsiella anguillimortifera. 
TABLE 1. Characteristics of Paracolobactrum anguillimortiferum compared with those of Edwardsiella tarda

\begin{tabular}{|c|c|c|c|c|c|}
\hline \multirow{2}{*}{ Test or substrate } & \multirow{2}{*}{$\begin{array}{l}\text { Paracolo- } \\
\text { bactrum } \\
\text { anguilli- } \\
\text { mortiferum }\end{array}$} & \multicolumn{3}{|c|}{ Edwardsiella tarda ${ }^{b}$} & \multirow{2}{*}{$\begin{array}{c}\text { Type strain } \\
\text { of } E . \text { tarda } \\
\text { (ATCC } \\
15947 \text { ) }\end{array}$} \\
\hline & & Reaction & $\%+$ & $\%(+)$ & \\
\hline 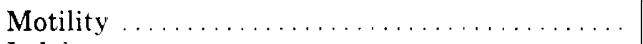 & + & + & 100 & \multirow{11}{*}{1.9} & + \\
\hline$\ldots \ldots \ldots \ldots \ldots \ldots \ldots \ldots \ldots \ldots$ & + & + & 98.1 & & + \\
\hline$\ldots \ldots \ldots \ldots \ldots \ldots \ldots \ldots$ & + & + & 100 & & + \\
\hline$\ldots \ldots \ldots \ldots \ldots \ldots \ldots \ldots$ & - & - & 0 & & - \\
\hline Citrate (Simmons') .... & - & - & 0 & & - \\
\hline Hydrogen sulfide (TSI) $\ldots \ldots \ldots \ldots \ldots \ldots \ldots$ & + & + & 97.5 & & + \\
\hline Nitrate to nitrite $\ldots \ldots \ldots \ldots \ldots \ldots \ldots$ & + & + & 100 & & + \\
\hline Gelatin liquefaction $\ldots \ldots \ldots \ldots \ldots \ldots \ldots$ & - & - & 0 & & - \\
\hline Urease (Christensen) $\ldots \ldots \ldots \ldots \ldots \ldots \ldots$ & - & - & 0 & & - \\
\hline Phenylalanine deaminase $\ldots \ldots \ldots \ldots \ldots \ldots$ & - & - & 0 & & - \\
\hline Lysine decarboxylase $\ldots \ldots \ldots \ldots \ldots \ldots \ldots$ & - & + & 100 & & + \\
\hline Ornithine decarboxylase $\ldots \ldots \ldots \ldots \ldots \ldots$ & - & + & 99.1 & \multirow[t]{6}{*}{0.4} & + \\
\hline Arginine dihydrolase $\ldots \ldots \ldots \ldots \ldots \ldots \ldots$ & - & - & 0 & & - \\
\hline 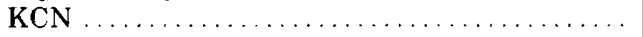 & - & - & 0 & & - \\
\hline Malonate $\ldots \ldots \ldots \ldots \ldots \ldots \ldots \ldots$ & - & - & 0 & & - \\
\hline Esculine hydrolysis $\ldots \ldots \ldots \ldots \ldots \ldots \ldots \ldots$ & - & - & 0 & & - \\
\hline \multicolumn{5}{|l|}{ Fermentation } & \\
\hline \multicolumn{6}{|l|}{ Glucose } \\
\hline Acid . & + & + & 100 & \multirow{9}{*}{1.1} & + \\
\hline Gas $\ldots \ldots \ldots \ldots \ldots \ldots \ldots \ldots \ldots \ldots$ & + & + & 97.5 & & + \\
\hline 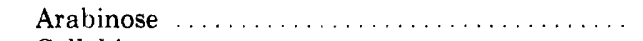 & - & - & 4.0 & & - \\
\hline Cellobiose $\ldots \ldots \ldots \ldots \ldots \ldots \ldots \ldots \ldots \ldots$ & - & - & 0 & & - \\
\hline Lactose $\ldots \ldots \ldots \ldots \ldots \ldots \ldots \ldots$ & - & - & 0 & & - \\
\hline 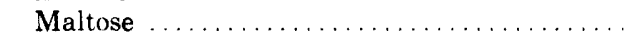 & + & + & 100 & & + \\
\hline 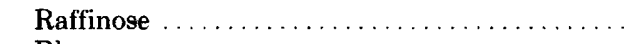 & - & - & 0 & & - \\
\hline 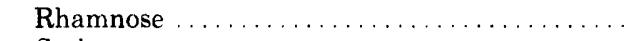 & - & - & 0 & & - \\
\hline 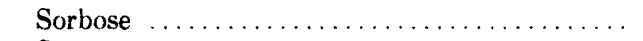 & - & - & 0 & & - \\
\hline Sucrose $\ldots \ldots \ldots \ldots \ldots \ldots \ldots \ldots \ldots \ldots$ & - & - & 0 & \multirow[t]{7}{*}{2.9} & - \\
\hline Trehalose $\ldots \ldots \ldots \ldots \ldots \ldots \ldots \ldots \ldots \ldots$ & - & - & 0 & & - \\
\hline 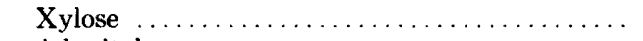 & - & - & 0 & & - \\
\hline Adonitol $\ldots \ldots \ldots \ldots \ldots \ldots \ldots \ldots \ldots \ldots \ldots \ldots \ldots \ldots \ldots \ldots$ & - & - & 0 & & - \\
\hline Dulcitol $\ldots \ldots \ldots \ldots \ldots \ldots \ldots \ldots \ldots$ & - & - & 0 & & - \\
\hline Erythritol $\ldots \ldots \ldots \ldots \ldots \ldots \ldots \ldots \ldots$ & - & - & 0 & & - \\
\hline 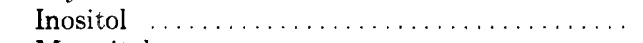 & - & - & 0 & & - \\
\hline 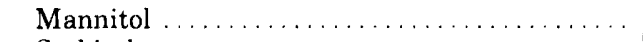 & - & - & 0 & 1.1 & - \\
\hline Sorbitol $\ldots \ldots \ldots \ldots \ldots \ldots \ldots \ldots \ldots \ldots \ldots \ldots \ldots \ldots \ldots \ldots$ & - & - & 0 & 0.1 & - \\
\hline Salicin $\ldots \ldots \ldots \ldots \ldots \ldots \ldots \ldots \ldots$ & - & - & 0 & 1.3 & - \\
\hline $\mathrm{ONPG}^{d} \quad \ldots \ldots \ldots \ldots \ldots \ldots \ldots \ldots \ldots \ldots \ldots \ldots \ldots \ldots \ldots \ldots$ & - & - & 0 & & \\
\hline
\end{tabular}

${ }^{a}$ Based on the description by Hoshina (1962).

${ }^{b}$ Based on a study of 800 strains of Edwardsiella tarda (Sakazaki and Tamura, unpublished data). +, Positive within 1 or 2 days of incubation; $(+)$, positive after 3 to 7 days; --, no reaction within 7 days; $\bullet$, no description.

"Designated as the neotype strain of Edwardsiella anguillimortifera.

a $o$-Nitrophenyl- $\beta$-D-galactopyranoside.

\section{LITERATURE CITED}

1. Ewing, W. H., A. C. McWhorter, M. R. Escobar, and A. H. Lubin. 1965. Edwardsiella, a new genus of Enterobacteriaceae based on a new species, $E$. tarda. Int. Bull. Bacteriol. Nomencl. Taxon. 15:33-38.

2. Hoshina, T. 1962. On a new bacterium, Paracolobactrum anguillimortiferum n. sp. Bull. Jpn. Soc. Sci. Fish.
28:162-164.

3. King, B. M., and D. L. Adler. 1964. A previously undescribed group of Enterobacteriaceae. Am. J. Clin. Pathol. 4:230-232.

4. Sakazaki, R., and Y. Murata. 1962. The new group of the Enterobacteriaceae, the Asakusa group. (In Japanese) Jpn. J. Bacteriol. 17:617-618. 